\title{
Yeast Securin is trafficked through the endomembrane system to regulate cell cycle arrest during the DNA damage response
}

\author{
Brenda R. Lemos, David P. Waterman1, and James E. Haber* \\ Department of Biology and Rosenstiel Basic Medical Sciences Research Center, \\ Brandeis University, Waltham MA 02454-9110
}

1. Current address: The Koch Institute for Integrative Cancer Research at Massachusetts Institute of Technology, 500 Main Street, Cambridge MA 02142

* To whom correspondence should be addressed 


\begin{abstract}
The yeast securin protein, Pds1, belongs to a class of highly conserved eukaryotic proteins that regulate the timing of chromatid segregation during mitosis by inhibiting separase, Esp1. During the metaphase to anaphase transition Pds1 is degraded by the ubiquitin-proteasome system in the nucleus, unleashing Esp1's protease activity to cleave cohesin surrounding sister chromatids. In response to DNA damage Pds1 is phosphorylated and stabilized, stalling mitotic progression to preserve genomic integrity. In addition, during the DNA damage checkpoint response, securin and separase are partially localized in the vacuole. Here we genetically dissect the requirements for securin's vacuolar localization and find that it is dependent on the Alkaline Phosphatase (ALP) endosomal transport pathway but not autophagy. Blocking retrograde traffic between the Golgi and the endoplasmic reticulum, by inhibiting COPI vesicle transport, drives Pds1 into the vacuole, whereas inhibiting antegrade transport by disrupting COPII-mediated traffic, results in the unexpected loss of Pds1 and the extinction of DNA damage-induced cell cycle arrest. We report that the induction of ER stress, either genetically or by treating cells with dithiothreitol, is sufficient to extinguish the DNA damage checkpoint, suggesting crosstalk between these two pathways. These data highlight new ways in which Pds1 and Esp1 are regulated during a DNA damage induced G2/M arrest and its requirement in the maintenance of the DNA damage checkpoint response.
\end{abstract}

\title{
Introduction
}

A single irreparable double strand break (DSB) activates the DNA damage checkpoint response in budding yeast. The DNA damage response is mediated by two PI3K-like kinases, Mec1 and Tel1 (ATR and ATM in mammals) that in turn phosphorylate several transducer and effector proteins, all to ensure G2/M arrest. Arrest prior to anaphase prevents cells from 
completing mitosis with broken chromosomes, allowing time for repair to take place $(1,2)$.

Failure to activate the DNA damage checkpoint response can result in genomic instability (3) and in higher eukaryotes may lead to disease (4).

In Saccharomyces cerevisiae, Pds1(securin) binds to Esp1(separase) during anaphase onset and together are trafficked to the nucleus where the Anaphase Promoting Complex (APC), along with its cofactor Cdc20, ubiquitinates Pds1, which is then destroyed by the ubiquitinproteasome system (5-8). Degradation of Pds1 allows Esp1 to cleave the Scc1 subunit of cohesin, allowing sister chromosome separation and the completion of mitosis $(9,10)$. In the presence of even a single DSB the DNA damage response is activated; Pds1 is phosphorylated and stabilized by the Chk1 kinase, which prevents Pds1's ubiquitination and thus blocks Esp1's protease activity and maintains G2/M arrest (11-13).

Cells suffering a single irreparable DSB arrest for 12-15 h, after which they turn off the DNA damage checkpoint and re-enter mitosis in a process termed adaptation (14-17). Several adaptation-defective mutations have been identified that render cells incapable of adapting and lead to permanent G2/M arrest (2). Why cells undergo adaptation in the presence of DNA damage is unknown, but this phenomenon is not limited to budding yeast. Indeed, adaptation has been described in both Xenopus egg extracts and in mammalian cells $(18,19)$. Adaptation thus allows cells with unrepaired DNA damage to divide and enhances the risk of genome instability. Therefore, it is of general interest to determine what mutations and conditions renders cells adaptation-defective when faced with irreparable DNA damage.

One set of adaptation-defective mutants that have been previously characterized are the deletions of Vps51 and other proteins belonging to the Golgi-associated retrograde protein complex (GARP) (14). Whereas Pds1-GFP normally localizes to the nucleus during DNA 
damage-induced G2/M arrest, in vps51 $\Delta$ cells Pds1-GFP instead largely forms puncta in the cytoplasm. Both the localization and adaptation defects were rescued by deleting the vacuolar protease Pep4 (14), which is also required for the maturation of other resident vacuolar proteases $(20,21)$. These observations along with the identification of a DNA damage-induced autophagy pathway termed, Genotoxin- induced Autophagy (GTA) (22), led us to suspect that Pds1 was a target of GTA. Here we show that Pds1 is targeted to the vacuole independently of autophagy and instead requires components of the alkaline phosphatase transport pathway (ALP) (23). Inhibiting Pds 1's vacuolar localization shortens cell arrest after the DNA damage checkpoint response is triggered, causing cells to resume mitosis more quickly in the presence of an unrepaired DSB. Additionally, we find a role for the major nuclear exporter Crm1 (Xpo1) in exporting Pds1 during the DNA damage checkpoint response and during valproic acid (VPA)induced nuclear delocalization.

The dynamic movements of Separase and Securin are evident by interfering with vesicle transport. When COPI vesicles are inhibited and retrograde traffic is blocked, Pds1 is more strongly driven into the vacuole. Conversely, upon conditional inhibition of COPII vesicles to block antegrade traffic, Pds1 is unexpectedly degraded and the DNA damage checkpoint response is turned off. This result is reproduced by treatment of cells with dithiothreotol (DTT) that also induces ER stress (24). These results suggest that induction of ER stress takes precedence over the DNA damage response. Taken together our results indicate that Pds1 is highly dynamic during DNA damage induced G2/M arrest, offering insights into a key regulator of the DNA damage checkpoint response.

\section{Results}

Pds1 Localizes to the vacuole independently of autophagy 
We have previously characterized an Mec1- and Tel1-dependent pathway for DNA damage-induced autophagy (22). We speculated that Pds1 is a target of GTA since we had shown Pds1 localized to vacuoles following a DSB. Since globular GFP is mostly resistant to vacuolar proteases, targets of autophagy can be monitored by fusing GFP to proteins and monitoring the appearance of GFP in the vacuole (25-27). However, GFP-tagged Pds1 in wild type cells was only seen localizing to the nucleus during DNA damage induced G2/M arrest but when the vacuolar protease, PEP4, was deleted, Pds1-GFP was visible in the vacuole (14) (Fig 1A). PEP4 encodes for proteinase $\mathrm{A}$ that is required for the activation of other vacuolar proteases proteinase B and C, $(28,29)$. In the absence of Pep4, protein degradation is reduced by $20-30 \%$, causing an accumulation of proteins in the vacuole and allowing visualization of GFP-tagged proteins otherwise not visible (30). Under these conditions Esp1-mCherry (Separase) also localizes to vacuoles, colocalizing with Pds1-GFP (Fig. 1B). This vacuolar localization allows us to study the genetic requirements for Pds1's vacuolar degradation.

Reasoning that autophagy is a likely pathway by which Pds1 is delivered to the vacuole during the DNA damage response, we deleted core autophagy proteins Atg1 and Atg13, which are both required for autophagic induction in yeast <ref>, in the pep $4 \Delta$ background and monitored Pds1-mCherry localization 6 h after HO induction. Surprisingly, Pds1 still localized to the vacuole in the absence of these core autophagy proteins (Fig. 1C). Additionally, we monitored Pds1 abundance during the DNA damage response and observed a 5-fold increase in Pds1 protein levels, peaking at $3 \mathrm{~h}$ after $\mathrm{HO}$ induction and falling back to initial levels by $12 \mathrm{~h}$ 
(Fig. 1D). This profile was unchanged in atgl $\Delta$ cells (Fig. 1D). We therefore conclude that

Pds1's vacuolar localization does not require autophagy.

\section{Pds1 vacuolar localization requires the ALP pathway}

We next extended our analysis to encompass other vacuolar-targeting pathways. The CPY pathway delivers vacuolar hydrolases and proteins from the Golgi to the vacuole through a pre-endosomal/pre-vacuolar compartment (PVC). The ALP pathway (alkaline phosphatase) delivers proteins directly from the Golgi to the vacuole. To test whether Pds 1 is delivered to the vacuole via the CPY pathway, we monitored its localization in a pep12 $\Delta$ mutant, which lacks a tSNARE involved in vesicle fusion with the late endosome (31). Pds1 vacuolar localization persisted in pep $4 \Delta$ cells lacking without Pep12, as judged by overlapping GFP signal with the vacuolar lumen stain CMAC (Fig. 2D). This was also the case in cells lacking the sorting receptor Pep1 that is also required for the CPY pathway (Fig. 2D) (32). Thus, Pds1 is not delivered to the vacuole via the CPY pathway.

We then examined the ALP pathway. The ALP pathway requires the heterotetrametric AP-3 adaptor complex to deliver vesicles from the Golgi to the vacuole (33). We monitored Pds1's localization in apm3 $\Delta$ cells, which lack the $\mu 3$ a subunit of AP-3 (34), after induction of a single HO-mediated DSB and found that Pds1 no longer localized to the vacuole in this mutant (Fig. 2A). To substantiate this finding we deleted the vacuolar kinase Yck3 required for the fusion of ALP vesicles with the vacuole (35). Pds1's vacuolar localization was also blocked in $y c k 3 \Delta$ cells (Fig. 2B). Taken together, these results suggest that Pds1 localizes to the vacuole using components of the ALP pathway. 


\section{apm $3 \Delta$ and yck3 $\Delta$ cells adapt faster after DNA damage}

We then asked how the ALP mutations would affect cell cycle arrest after DNA damage, in particular how cells would arrest and adapt after induction of a single unrepaired DSB. Cells were grown overnight in YEP supplemented with 3\% lactic acid and plated on YEP plates containing $2 \%$ galactose, which induces a single DSB created by HO endonuclease (17). G1 cells were micromanipulated and monitored for $24 \mathrm{~h}$ to determine the time at which cells adapted. WT cells arrest at G2/M for 12-15 h then turn off the DNA damage checkpoint and resume mitosis (17) (Fig. 2C). Although only $25 \%$ of WT cells had adapted by $12 \mathrm{~h}$, over $50 \%$ of apm3 3 and $y c k 3 \Delta$ cells had adapted (Fig. 2C). These data suggest that Pds1's vacuolar localization is required for proper maintenance of G2/M arrest after DNA damage.

\section{Pds1 localizes to the vacuole in the absence of COPI vesicles}

Given that the ALP pathway in responsible for Pds1's localization to the vacuole during the DNA damage response, we reasoned that perturbing other cell endomembrane trafficking components may alter Pds1's localization. To this end we examined the role of COPI vesicles, which are a coatomer proteins that deliver vesicles from the cis-Golgi to the ER, with the rationale that if Pds1 localizes to the vacuole during the DNA damage response, it may also be trafficked back to the nucleus via retrograde trafficking (36). Therefore, if retrograde trafficking is blocked, Pds1 might accumulate in the vacuole. We tagged the delta subunit of COPI vesicles, Ret2 (37), with an auxin-inducible degron (AID) $(38,39)$. Incorporation of AID at the Cterminus of Ret 2 and degradation of the Ret 2 subunit upon auxin treatment was confirmed via western blot (Fig. S1). Cultures were grown overnight to exponential phase and galactose was added to induce a DSB for $2 \mathrm{~h}$ prior to auxin treatment. Fewer than $25 \%$ of cells had Pds 1 in the vacuole before IAA treatment whereas over $75 \%$ of cells had Pds 1 in the vacuole in the absence 
of COPI vesicles, even in the presence of Pep4 (Fig. 3A). These results suggest that in the absence of COPI vesicles, Pds1 is cycled into the vacuole and fails to be retrograde transported back toward the nucleus.

\section{Inhibition of COPII vesicles leads to degradation of Pds1 and inactivation of the DNA damage checkpoint response}

Since Pds1 travels to the vacuole via the ALP pathway, we presumed that it undergoes anterograde traffic from the ER to the Golgi and therefore might also depend on COPII vesicle transport. We tested whether conditionally inhibiting COPII vesicles via Sec23-AID depletion altered Pds1's vacuolar localization. Cells were grown overnight in YEP-Lac to exponential phase and treated with $2 \%$ galactose to induce a DSB. $2 \mathrm{~h}$ after DSB induction, auxin was added to cultures to deplete Sec23. In pep $4 \Delta$ cells treated only with galactose, Pds1 localized to both the vacuole and nucleus. As expected, in cells only treated with galactose to induce a DSB, Pds 1 accumulated in the cells during arrest, with protein concentration peaking around $6 \mathrm{~h}$ after induction of a DSB and falling thereafter (Fig. 3B). Surprisingly, in cells depleted of Sec23, beginning $2 \mathrm{~h}$ after induction of a DSB, Pds1 levels were much lower and sometimes undectable (Fig. 3B). Moreover, the Rad53 kinase, whose phosphorylation is indicative of checkpoint activation, was completely dephosphorylated (Fig. 3B). These surprising results suggest that disrupting anterograde traffic by inhibiting COPII vesicles destabilized Pds1 and blocked the maintenance of the DNA damage response.

\section{DTT treatment after a DSB turns off the DNA damage response}

Since COPII vesicles originate in the ER, we considered that degradation of Sec23 and inhibition of COPII vesicles leads to the induction of the ER-stress response, including the unfolded protein response (Walter and Ron, 2011; Jonikas et al. 2009). If this were the case, our 
data would suggest that the ER-stress response bypasses the DNA damage response, and that both the DNA damage response and the ER-stress response cannot signal simultaneously. To address whether this was indeed the case, we treated cells experiencing a DSB with dithiothreitol (DTT). DTT is a strong reducing agent that blocks the formation of disulfide bonds and leads to the accumulation of unfolded proteins in the ER, activating the unfolded protein response (UPR) and the ER-stress response $(24,40)$. Cells were grown overnight in YEP-Lac to exponential phase and then treated with $2 \%$ galactose to induce a DSB. Cultures were split $2 \mathrm{~h}$ after the addition of galactose and one half was treated with $2 \mathrm{mM}$ of DTT. Cells were collected at various time-points to monitor levels of Pds1 and the activation of the DNA damage checkpoint via Rad53 phosphorylation. Interestingly, cells experiencing a DSB and treated with DTT had much lower levels of Pds1 while Rad53 became fully dephosphorylated, compared to cells treated with galactose only, indicative of an inactive DNA damage checkpoint response (Fig. 4A). Moreover, addition of $2 \mathrm{mM}$ DTT $6 \mathrm{~h}$ after creating the DSB also released most cells from G2/M arrest after induction of a single HO endonuclease-induced DSB (Fig. 4B). These results suggest that after DTT treatment and activation of the UPR, the DNA damage response is turned off even in the presence of a an irreparable DSB, again suggesting that both the UPR and the DNA damage response cannot be active at the same time.

\section{Pds1 is required for the maintenance of the DNA damage checkpoint response}

Our results indicate that both inhibition of COPII vesicle transport and DTT treatment lead to the degradation of Pds1 and inactivation of the DNA damage checkpoint response. However, we do not know whether these observations reflect a direct role for Pds1 in maintaining the DNA damage response versus simply acting as an effector. To address this question, we tagged Pds1 with AID to enable the degradation of Pds1 after the activation of the DNA damage checkpoint. 
Cells were grown overnight in YEP-Lac and 2\% galactose was added to induce a DSB. $4 \mathrm{~h}$ after galactose treatment, cultures were split and $500 \mathrm{uM}$ of auxin was added to degrade Pds1. Pds1 levels and Rad53 phosphorylation were monitored via western blot. In galactose-only treated cells at 4 h, Pds 1 protein levels accumulate while Rad53 is phosphorylated, indicative of an active DNA damage checkpoint response (Fig. 4C). In the presence of auxin, Pds1 was rapidly degraded, coinciding with Rad53 dephosphorylation indicating that Pds1 is indeed required for the maintenance of the DNA damage response (Fig. 4C). We suspect that degradation of Pds1 enables Esp1 to cleave sister cohesin rings and allows cell division to proceed, and that progression of the cells from metaphase to anaphase switches off DNA damage signaling.

\section{Crm1 is an exporter of Pds1 during the DNA damage response}

We then asked how Pds1 was exported from the nucleus during DNA damage. Crm1 (Xpo1) is a major exporter in eukaryotic cells $(41,42)$. Because Crm1 is essential, we again used the AID system and tagged Crm1 with an AID tag tag at the C-terminus to degrade the protein upon IAA addition. Again, cells were grown overnight in YEP-Lac to exponential phase and $1 \mathrm{~h}$ prior to galactose induction of a single DSB, we added $500 \mu \mathrm{M}$ of IAA to promote Crm1 degradation. We monitored Pds 1 levels in the absence of $\mathrm{Crm} 1$ for a $24 \mathrm{~h}$ period in the presence of a single DSB. In the absence of Crm1, we observed higher overall levels of Pds1 during the DNA damage response (Fig. 5D) suggesting that Crm1 is important in displacing Pds1 out of the nucleus during the DNA damage checkpoint response.

To examine further the role of Crm1, we took advantage of the observation that some proteins were dramatically exported from the nucleus after DNA damage after treatment with the histone deacetylase inhibitor, valproic acid (VPA) $(43,44)$. After inducing a single DSB and allowing cells $6 \mathrm{~h}$ to become G2/M arrested we added $10 \mu \mathrm{M}$ of VPA and Pds 1-GFP localization 
was monitored using fluorescence microscopy. Pds1 localizes to the nucleus during the DNA damage-induced G2/M arrest; however, 30 min after VPA treatment Pds1 rapidly localized to the cytoplasm in puncta-like structures (Fig. 5B). To monitor Pds1 levels after VPA treatment we performed a time-course experiment and quantified Pds1 using western blot analysis. As we have previously shown in this study, Pds1 levels peaked around $6 \mathrm{~h}$, after which levels began to slowly decrease. However, treating cells with VPA significantly reduced Pds 1 levels during the DNA damage response (Fig. 5A) In contrast, in Crm1-AID cells treated with IAA prior to start of experiment (Fig. 5A), Pds1 remained in the nucleus for an extended time (Fig. 5B). Very few cells had cytoplasmic Pds1 signal prior to VPA treatment, in both the presence or the absence of Crm1 (Fig. 5C), but in the absence of Crm1 after VPA treatment, Pds1 accumulates in the vacuole, even in the presence of the vacuolar protease Pep4.

\section{Discussion}

This study began with the observation that Pds1-GFP could be seen in the vacuole after DSB damage, in the absence of the vacuolar protease PEP4 (14). Our initial hypothesis, that Pds1 is targeted to the vacuole in an autophagy dependent manner, proved incorrect, since Pds 1 localizes to vacuoles after DSB induction in the absence of core autophagy kinase, Atg1 and regulatory protein Atg13. Instead, we determined that both Pds1 and Esp1 are targeted to the vacuole using the ALP pathway. Although the ALP pathway is named because of the substrate initially described in this pathway (alkaline phosphatase), other proteins such as the vacuolar amino acid transporters Ypq1, Ypq2 and Ypq3 also require components of this pathway (45). We found that inhibition of the ALP pathway causes cells to adapt faster in response to a persistent DSB. This result suggests that proper cell cycle arrest during the DNA damage checkpoint is enhanced by the vacuolar degradation of Pds1. This conclusion is also supported by degrading 
the nuclear exporter Crm1, since we observe higher levels of Pds1 in its absence. Taken together, these results suggest that proper regulation of Pds1 during the DNA damage response requires nuclear export and traffic to the vacuole. This conclusion is supported by our discovery that inhibition of COPI vesicles significantly affected the localization of Pds1, which now accumulated in the vacuole. These results favor a model where Golgi-accumulated Pds1 gets delivered to the vacuole via AP-3 coated vesicles. This model also supports previous observations that Pds1 is mislocalized in the absence of components of the GARP complex (14). GARP mediates the vesicle transfer from endosomes in the secretory pathway to the trans-Golgi network $(46,47)$. We suggest that in the absence of GARP, Pds1 is trapped in endosomes and is incapable of re-localizing to the endocytic pathway and back into the nucleus. However, the defects observed in GARP mutants could be rescued either by deleting PEP4 or by fusing Esp1 with the nuclear localization signal of SV40 to drive Esp1 into the nucleus without relying on its normal chaperone, Pds1 (14).

Many proteins required for protein transport interact with cargo via conserved motifs. Indeed, analysis of Pds1's protein sequence identifies various protein motifs that are consistent with the export and trafficking pathways in our data. For example, within Pds 1 we identify amino acid sequence 112-126 that is consistent with a nuclear export signal (NES) for Crm1. There are also multiple amino acid motifs that have been associated with binding to the mu subunit of the adaptor protein complex AP-3 (48), and while Pds1 has three different Apm3 binding motifs, Esp1 has twenty. Furthermore, both Esp1 and Pds1 have clathrin binding motifs $(49,50)$ that are consistent with COPII vesicle transport. We have yet to determine whether Pds1 or Esp1 directly interact with vesicle transport proteins to mediate its traffic. Given that Esp1 has a 1630 aa sequence while Pds1 has only 373 aa, Esp1's larger surface area creates more 
opportunity for other protein-protein interactions. Pds1 is highly unstructured, but when bound to Esp1 they together form an elongated shape. However a crystal structure of Pds1 bound to Esp1 only resolved residues $258-373$ bound to Esp1's protease site, leaving open the possibility that the C-terminal of Pds1 may have flexibility to interact with other proteins while still inhibiting Esp1's activity (51).

While looking for proteins that affect Pds1's localization during the DNA damage response we found that inhibition of COPII vesicles stimulates degradation of Pds1 and dephosphorylation of Rad53. Similar results were observed when cells were treated with DTT, which has been shown to induce the UPR. These results suggest that COPII inhibition during the DNA damage response induces ER stress and that the UPR is dominant over the DNA damage response. Although this crosstalk has not been explored in budding yeast, a similar connection has been made in mammalian cells, where UPR induction after DNA damage prevents the expression of p21 during DNA damage and increases p53's genotoxic-induced apoptosis (52). Additionally, tunicamycin-induced ER stress has been shown to decrease DNA repair in tumor cells by stimulating Rad51 degradation (53). We have yet to explore the effects of the UPR in DNA repair.

Taken together our results show that Pds1 is highly dynamic in cells during the DNA damage response. Until recently, Pds1 was thought to only localize to the nucleus and only function to regulate Esp1 activity during the cell cycle. Here we show that Pds1 and Esp1 also localize to the vacuole, cytoplasm and Golgi. This multi-compartment localization has been already observed in mammalian cells; in fractionated mammalian cell lysates, securin is found in nuclear, ER, Golgi, and cytoplasmic fractions (54). We have not ruled out the possibility that securin has additional roles outside of the nucleus during the DNA damage response; however, 
the fact that Esp1 colocalizes with Pds1 in the vacuole during the DNA damage response makes it likely that Pds1 still functions outside of the nucleus to control Esp1's localization, and to maintain G2/M arrest.

\section{Materials and Methods}

\section{Media, strains and adaptation assays}

All strains used in this study are derivatives of JKM179 (55). All mutant strains were created using one-step PCR homology cassette amplification with lithium acetate transformation procedure $(56,57)$. AID-tagged strains were constructed as previously described (39). For timecourse and imaging experiments, cells were grown overnight in YEP-Lac to early exponentialphase. $2 \%$ galactose was added to cultures to induce expression of HO endonuclease. For all AID tagged experiments, $500 \mu \mathrm{M}$ of 3-Indoleacetic acid (IAA) (Sigma Aldrich) was added to cultures $2 \mathrm{~h}$ after galactose addition. Adaptation assays were carried out as previously described $(17,58)$. Briefly, cells were grown overnight in YEP-Lac and plated in YEP-Lac agarose plates containing $2 \%$ galactose. $50 \mathrm{G} 1$ cells were micro-manipulated and monitored for arrest and adaptation, results shown are a replicate of at least three different adaptation study.

\section{Western Blotting}

$50 \mathrm{ml}$ of cells from early exponential-phase cultures (approximately 1 x 106 cells $/ \mathrm{ml}$ ) were harvested for each time-point within a time-course experiment and cell pellets were processed using the trichloroacetic acid (TCA) protocol previously described (59). 10\%, 8\% and 6\% Polyacrylamide gels were used in these studies. Blotting was performed using anti-PGK1 (Abcam, ab113687), anti-MYC (Abcam, 9E10), anti-HA (Abcam, HA.C5) and anti-Rad53 (Abcam, ab166859). Blots were imaged using the ChemiDoc Imager (BioRad) and analyzed 
using the Image Lab (BioRad) software. Protein quantifications were carried out by normalizing protein intensities to Pgk1 loading controls.

\section{Image Acquisition and Analysis}

Cells were grown overnight in YEP-Lac and 2\% galactose was added to induce expression of HO. Cells were harvested $6 \mathrm{~h}$ after galactose addition and washed in synthetic complete medium before imaging. A Nikon Eclipse E600 microscope was used for imaging. Images were processed and analyzed using the FIJI software. Student's t-test with a Sidak-Bonferroni correction was performed using GraphPad Prism. The Eukaryotic Linear Motif (ELM) resource (http://elm.eu.org/index.html) was used to analyze the protein sequence of Pds1. The ELM prediction tool scans the protein sequence for motifs corresponding to experimentally validated protein motifs (60).

\section{Acknowledgements}

We are grateful to Eric Baehrecke and Susan Ferro-Novick for their comments and suggestions on the manuscript. This work was supported by grants GM61766 and R35GM127029. B.L. and D.P.W. were both Trainees of NIH Genetics Training Grant TM32 GM007122. B.L. also received

a Research Supplement to Promote Diversity in Health-Related Research for grant R35GM127029. 


\section{Figure legends}

Figure 1: Pds 1 localizes to vacuoles independently of autophagy

A) Pds1-GFP localization in cells experiencing a single HO induced DSB. In the absence of the vacuolar protease Pep4, Pds1 localizes to the vacuole. FM4-64 highlights the vacuolar membrane. B) Esp1-mCherry co-localizes with Pds1-GFP in the vacuole. C) Pds1-mCherry localization in the absence of the core autophagy kinase Atg1. D) Quantification of Pds1 levels in cells experiencing a single HO-induced DSB in JKM179 background and in atg1s cells.

Figure 2: Pds1 localizes to vacuoles using components of the ALP pathway A) Pds1-GFP localizes to vacuoles in pep $4 \Delta$ but localization is blocked in pep $4 \Delta$ apm $3 \Delta$ cells b) In the absence of the vacuolar kinase Yck3, Pds1-GFP does not localize to the vacuole. B) $24 \mathrm{~h}$ adaptation assay in WT, $y c k 3 \Delta$ and apm $3 \Delta$ cells. ${ }^{*} \mathrm{p}<0.005$ students t-test with Bonferroni correction from three separate experiments. C) Pds1-GFP localization in the absence of

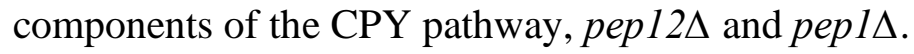

Figure 1: Pds1's localization and concentration in the absence of COPI and COPII vesicles A) Western blot showing degradation of COPI subunit Ret2-AID-MYC upon IAA treatment. Pds1-mCherry localization in the background of Ret2-AID with quantification of the fraction of cells with Pds1 in the vacuole before and after IAA treatment. B) Western blot and quantification (C) of Pds1 in the absence of the COPII subunit Sec23.

Figure 4: Effect of DTT on the DNA damage checkpoint A) Cells with a galactose-induced HO DSB at $\mathrm{t}=0$ were treated with $2 \mathrm{mM}$ DTT at $6 \mathrm{~h}$ and the abundance of Pds1-myc was monitored by Western blot. B) Release of cell cycle arrest when cells with an HO induced DSB were treated with $2 \mathrm{mM}$ DTT at 6 h. C) Loss of Rad53 phosphorylation after Pds1-MYC-AID was degraded by IAA treatment. 
Figure 5: Crm1 is an exporter of Pds1 during the DNA damage response

a) JKM179 cells expressing the F-box protein Tir1, Crm1-MYC-AID and Pds1-HA following HO induced DSB b) VPA treated cells in the presence of Crm1 or absence following IAA treatment c) Quantification of Pds1 localization in cells treated with VPA and IAA with VPA. Arrow shows vacuolar localization of Pds1.d) Quantification of Pds1 in cells suffering from HO induced DSB with and without $500 \mu \mathrm{M}$ of IAA normalized to Pgk1 loading control and Pds1 levels at $6 \mathrm{~h}$ in cells treated with vehicle only $(\mathrm{EtOH})$. 


\section{Table 1. Strains used in this study}

\begin{tabular}{|c|c|}
\hline JKM179 & $\begin{array}{l}\text { ho } \triangle \text { MATa hml }:: A D E 1 \text { hmr } \triangle:: A D E 1 \text { ade1-100 leu2-3,112 lys5 } \\
\text { trp1::his } G^{\prime} \text { ura3-52 ade3::GAL::HO }\end{array}$ \\
\hline DW184 & JKM179 ura3-52::pNHK53 (URA3, ADH1::TIR1) \\
\hline BL011 & DW184 CRM1-MYC-AID::KAN PDS1-GFP ::TRP1 \\
\hline BL021 & JKM179 PDS1-HA::URA3 atg1 $\because: \mathrm{HPH}$ \\
\hline BL024 & DW184 CRM1-MYC-AID::KAN PDS1-HA::TRP1 \\
\hline BL070 & JKM179 PDS1-HA::URA3 \\
\hline BL109 & $\begin{array}{l}\text { JKM179 pep } 4 \Delta:: T R P 1 \text { PDS1-mCherry::KAN } \\
\text { GFP-ATG8::URA3 }\end{array}$ \\
\hline BL108 & BL109 atg11 $1:: \mathrm{HPH}$ \\
\hline BL113 & BL109 $\operatorname{atg} 1 \Delta:: \mathrm{HPH}$ \\
\hline BL122 & JKM179 PDS1-HA::TRP1 \\
\hline BL123 & JKM179 PDS1-GFP::TRP1 \\
\hline BL168 & 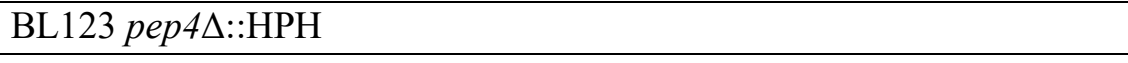 \\
\hline BL201 & BL168 ESP1-mCherry::KAN \\
\hline BL207 & BL168 pep1 $1:$ NAT \\
\hline BL209 & BL168 рер12 $\Delta::$ NAT \\
\hline BL214 & DW184 PDS1-GFP::TRP1 RET2-MYC-AID::HPH \\
\hline BL216 & 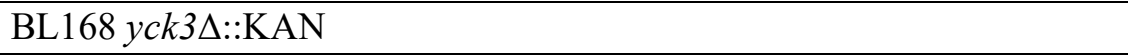 \\
\hline BL291 & BL214 HTB1-CFP::HPH \\
\hline BL311 & BL168 apm3 $\Delta:: \mathrm{KAN}$ \\
\hline BL342 & BL123 HTB1-mCherry::KAN \\
\hline BL346 & BL342 yck3 $\Delta:: \mathrm{KAN}$ \\
\hline BL348 & BL342 apm3s::KAN \\
\hline BL355 & $\begin{array}{l}\text { DW184 PDS1-GFP::TRP1 pep4A::HPH HTB1-mCherry::KAN SEC23 - } \\
\text { MYC-AID::NAT }\end{array}$ \\
\hline BL390 & DW184 SEC23::Sec23-MYC-AID::NAT PDS1-HA::KAN \\
\hline
\end{tabular}




\section{Literature cited:}

1. Zhou BB, Elledge SJ. The DNA damage response: putting checkpoints in perspective. Nature 2000;408(6811):433-439.

2. Harrison JC, Haber JE. Surviving the breakup: the DNA damage checkpoint. Annu Rev Genet 2006;40:209-235.

3. Tubbs A, Nussenzweig A. Endogenous DNA Damage as a Source of Genomic Instability in Cancer. Cell 2017;168(4):644-656.

4. Bartek J, Lukas J, Bartkova J. DNA damage response as an anti-cancer barrier: damage threshold and the concept of 'conditional haploinsufficiency'. Cell Cycle 2007;6(19):2344-2347.

5. Jensen S, Segal M, Clarke DJ, Reed SI. A novel role of the budding yeast separin Esp1 in anaphase spindle elongation: evidence that proper spindle association of Esp1 is regulated by Pds1. J Cell Biol 2001;152(1):27-40.

6. Agarwal R, Cohen-Fix O. Phosphorylation of the mitotic regulator Pds 1/securin by Cdc28 is required for efficient nuclear localization of Esp1/separase. Genes Dev 2002;16(11):1371-1382.

7. Morgan DO. Regulation of the APC and the exit from mitosis. Nat Cell Biol 1999;1(2):E47-53.

8. Fang G, Yu H, Kirschner MW. Direct binding of CDC20 protein family members activates the anaphase-promoting complex in mitosis and G1. Mol Cell 1998;2(2):163-171.

9. Uhlmann F, Lottspeich F, Nasmyth K. Sister-chromatid separation at anaphase onset is promoted by cleavage of the cohesin subunit Scc1. Nature 1999;400(6739):37-42.

10. Uhlmann F, Wernic D, Poupart MA, Koonin EV, Nasmyth K. Cleavage of cohesin by the CD clan protease separin triggers anaphase in yeast. Cell 2000;103(3):375-386.

11. Wang H, Liu D, Wang Y, Qin J, Elledge SJ. Pds1 phosphorylation in response to DNA damage is essential for its DNA damage checkpoint function. Genes Dev 2001;15(11):13611372.

12. Tinker-Kulberg RL, Morgan DO. Pds1 and Esp1 control both anaphase and mitotic exit in normal cells and after DNA damage. Genes Dev 1999;13(15):1936-1949.

13. Sanchez Y, Bachant J, Wang H, Hu F, Liu D, Tetzlaff M, Elledge SJ. Control of the DNA damage checkpoint by chk1 and rad53 protein kinases through distinct mechanisms. Science 1999;286(5442):1166-1171.

14. Dotiwala F, Eapen VV, Harrison JC, Arbel-Eden A, Ranade V, Yoshida S, Haber JE. DNA damage checkpoint triggers autophagy to regulate the initiation of anaphase. Proc Natl Acad Sci U S A 2013;110(1):E41-49. 
15. Sandell LL, Zakian VA. Loss of a yeast telomere: arrest, recovery, and chromosome loss. Cell 1993;75(4):729-739.

16. Toczyski DP, Galgoczy DJ, Hartwell LH. CDC5 and CKII control adaptation to the yeast DNA damage checkpoint. Cell 1997;90(6):1097-1106.

17. Lee SE, Moore JK, Holmes A, Umezu K, Kolodner RD, Haber JE. Saccharomyces Ku70, mre11/rad50 and RPA proteins regulate adaptation to G2/M arrest after DNA damage. Cell 1998;94(3):399-409.

18. Syljuasen RG, Jensen S, Bartek J, Lukas J. Adaptation to the ionizing radiation-induced G2 checkpoint occurs in human cells and depends on checkpoint kinase 1 and Polo-like kinase 1 kinases. Cancer Res 2006;66(21):10253-10257.

19. Yoo HY, Kumagai A, Shevchenko A, Shevchenko A, Dunphy WG. Adaptation of a DNA replication checkpoint response depends upon inactivation of Claspin by the Polo-like kinase. Cell 2004;117(5):575-588.

20. Zubenko GS, Park FJ, Jones EW. Mutations in PEP4 locus of Saccharomyces cerevisiae block final step in maturation of two vacuolar hydrolases. Proc Natl Acad Sci U S A 1983;80(2):510-514.

21. Wolff AM, Din N, Petersen JG. Vacuolar and extracellular maturation of Saccharomyces cerevisiae proteinase A. Yeast 1996;12(9):823-832.

22. Eapen VV, Waterman DP, Bernard A, Schiffmann N, Sayas E, Kamber R, Lemos B, Memisoglu G, Ang J, Mazella A, Chuartzman SG, Loewith RJ, Schuldiner M, Denic V, Klionsky DJ, et al. A pathway of targeted autophagy is induced by DNA damage in budding yeast. Proc Natl Acad Sci U S A 2017;114(7):E1158-E1167.

23. Stepp JD, Huang K, Lemmon SK. The yeast adaptor protein complex, AP-3, is essential for the efficient delivery of alkaline phosphatase by the alternate pathway to the vacuole. J Cell Biol 1997;139(7):1761-1774.

24. Schuck S, Gallagher CM, Walter P. ER-phagy mediates selective degradation of endoplasmic reticulum independently of the core autophagy machinery. J Cell Sci 2014;127(Pt 18):4078-4088.

25. Torggler R, Papinski D, Kraft C. Assays to Monitor Autophagy in Saccharomyces cerevisiae. Cells 2017;6(3).

26. Jones EW, Zubenko GS, Parker RR. PEP4 gene function is required for expression of several vacuolar hydrolases in Saccharomyces cerevisiae. Genetics 1982;102(4):665-677.

27. Ammerer G, Hunter CP, Rothman JH, Saari GC, Valls LA, Stevens TH. PEP4 gene of Saccharomyces cerevisiae encodes proteinase A, a vacuolar enzyme required for processing of vacuolar precursors. Mol Cell Biol 1986;6(7):2490-2499. 
28. Woolford CA, Daniels LB, Park FJ, Jones EW, Van Arsdell JN, Innis MA. The PEP4 gene encodes an aspartyl protease implicated in the posttranslational regulation of Saccharomyces cerevisiae vacuolar hydrolases. Mol Cell Biol 1986;6(7):2500-2510.

29. Winters CM, Chiang HL. Yeast as a Model System to Study Trafficking of Small Vesicles Carrying Signal-less Proteins In and Out of the Cell. Curr Protein Pept Sci 2016;17(8):808-820.

30. Hung GC, Brown CR, Wolfe AB, Liu J, Chiang HL. Degradation of the gluconeogenic enzymes fructose-1,6-bisphosphatase and malate dehydrogenase is mediated by distinct proteolytic pathways and signaling events. J Biol Chem 2004;279(47):49138-49150.

31. Gerrard SR, Mecklem AB, Stevens TH. The yeast endosomal t-SNARE, Pep12p, functions in the absence of its transmembrane domain. Traffic 2000;1(1):45-55.

32. Seaman MN, McCaffery JM, Emr SD. A membrane coat complex essential for endosome-to-Golgi retrograde transport in yeast. J Cell Biol 1998;142(3):665-681.

33. Feyder S, De Craene JO, Bar S, Bertazzi DL, Friant S. Membrane trafficking in the yeast Saccharomyces cerevisiae model. Int J Mol Sci 2015;16(1):1509-1525.

34. Cowles CR, Odorizzi G, Payne GS, Emr SD. The AP-3 adaptor complex is essential for cargo-selective transport to the yeast vacuole. Cell 1997;91(1):109-118.

35. Anand VC, Daboussi L, Lorenz TC, Payne GS. Genome-wide analysis of AP-3dependent protein transport in yeast. Mol Biol Cell 2009;20(5):1592-1604.

36. Bednarek SY, Ravazzola M, Hosobuchi M, Amherdt M, Perrelet A, Schekman R, Orci L. COPI- and COPII-coated vesicles bud directly from the endoplasmic reticulum in yeast. Cell 1995;83(7):1183-1196.

37. Cosson P, Demolliere C, Hennecke S, Duden R, Letourneur F. Delta- and zeta-COP, two coatomer subunits homologous to clathrin-associated proteins, are involved in ER retrieval. EMBO J 1996;15(8):1792-1798.

38. Nishimura K, Fukagawa T, Takisawa H, Kakimoto T, Kanemaki M. An auxin-based degron system for the rapid depletion of proteins in nonplant cells. Nat Methods 2009;6(12):917922.

39. Morawska M, Ulrich HD. An expanded tool kit for the auxin-inducible degron system in budding yeast. Yeast 2013;30(9):341-351.

40. Hetz C, Papa FR. The Unfolded Protein Response and Cell Fate Control. Mol Cell 2018;69(2):169-181.

41. Turner JG, Sullivan DM. CRM1-mediated nuclear export of proteins and drug resistance in cancer. Curr Med Chem 2008;15(26):2648-2655. 
42. Ishizawa J, Kojima K, Hail N, Jr., Tabe Y, Andreeff M. Expression, function, and targeting of the nuclear exporter chromosome region maintenance 1 (CRM1) protein. Pharmacol Ther 2015;153:25-35.

43. Robert T, Vanoli F, Chiolo I, Shubassi G, Bernstein KA, Rothstein R, Botrugno OA, Parazzoli D, Oldani A, Minucci S, Foiani M. HDACs link the DNA damage response, processing of double-strand breaks and autophagy. Nature 2011;471(7336):74-79.

44. Li Y, Seto E. HDACs and HDAC Inhibitors in Cancer Development and Therapy. Cold Spring Harb Perspect Med 2016;6(10).

45. Llinares E, Barry AO, Andre B. The AP-3 adaptor complex mediates sorting of yeast and mammalian PQ-loop-family basic amino acid transporters to the vacuolar/lysosomal membrane. Sci Rep 2015;5:16665.

46. Conibear E, Cleck JN, Stevens TH. Vps51p mediates the association of the GARP (Vps52/53/54) complex with the late Golgi t-SNARE Tlg1p. Mol Biol Cell 2003;14(4):16101623.

47. Conibear E, Stevens TH. Vps52p, Vps53p, and Vps54p form a novel multisubunit complex required for protein sorting at the yeast late Golgi. Mol Biol Cell 2000;11(1):305-323.

48. Darsow T, Burd CG, Emr SD. Acidic di-leucine motif essential for AP-3-dependent sorting and restriction of the functional specificity of the Vam3p vacuolar t-SNARE. J Cell Biol 1998;142(4):913-922.

49. Brett TJ, Traub LM, Fremont DH. Accessory protein recruitment motifs in clathrinmediated endocytosis. Structure 2002;10(6):797-809.

50. Popova NV, Deyev IE, Petrenko AG. Clathrin-mediated endocytosis and adaptor proteins. Acta Naturae 2013;5(3):62-73.

51. Luo S, Tong L. Molecular mechanism for the regulation of yeast separase by securin. Nature 2017;542(7640):255-259.

52. Mlynarczyk C, Fahraeus R. Endoplasmic reticulum stress sensitizes cells to DNA damage-induced apoptosis through p53-dependent suppression of p21(CDKN1A). Nat Commun 2014;5:5067.

53. Yamamori T, Meike S, Nagane M, Yasui H, Inanami O. ER stress suppresses DNA double-strand break repair and sensitizes tumor cells to ionizing radiation by stimulating proteasomal degradation of Rad51. FEBS Lett 2013;587(20):3348-3353.

54. Moreno-Mateos MA, Espina AG, Torres B, Gamez del Estal MM, Romero-Franco A, Rios RM, Pintor-Toro JA. PTTG1/securin modulates microtubule nucleation and cell migration. Mol Biol Cell 2011;22(22):4302-4311. 
55. Moore JK, Haber JE. Cell cycle and genetic requirements of two pathways of nonhomologous end-joining repair of double-strand breaks in Saccharomyces cerevisiae. Mol Cell Biol 1996;16(5):2164-2173.

56. Wach A, Brachat A, Pohlmann R, Philippsen P. New heterologous modules for classical or PCR-based gene disruptions in Saccharomyces cerevisiae. Yeast 1994;10(13):1793-1808.

57. Amberg DC, Burke DJ, Strathern JN. High-efficiency transformation of yeast. CSH Protoc 2006;2006(1).

58. Waterman DP, Zhou F, Li K, Lee CS, Tsabar M, Eapen VV, Mazzella A, Haber JE. Live cell monitoring of double strand breaks in S. cerevisiae. PLoS Genet 2019;15(3):e1008001.

59. Pellicioli A, Lee SE, Lucca C, Foiani M, Haber JE. Regulation of Saccharomyces Rad53 checkpoint kinase during adaptation from DNA damage-induced G2/M arrest. Mol Cell 2001;7(2):293-300.

60. Gouw M, Michael S, Samano-Sanchez H, Kumar M, Zeke A, Lang B, Bely B, Chemes LB, Davey NE, Deng Z, Diella F, Gurth CM, Huber AK, Kleinsorg S, Schlegel LS, et al. The eukaryotic linear motif resource - 2018 update. Nucleic Acids Res 2018;46(D1):D428-D434. 
bioRxiv preprint doi: https://doi.org/10.1101/2019.12.15.876896; this version posted December 15, 2019. The copyright holder for this preprint (which was not certified by peer review) is the author/funder, who has granted bioRxiv a license to display the preprint in perpetuity. It is made available under aCC-BY-NC-ND 4.0 International license.

\section{Supplemental Information}

\section{Supplemental Figure 1:}

JKM179 cells expressing the F-box protein Tir1 and Ret2-MYC-AID; Ret2 is degraded after IAA treatment, persisting 20 hs after IAA treatment. 
bioRxiv preprint doi: https://doi.org/10.1101/2019.12.15.876896; this version posted December 15, 2019. The copyright holder for this preprint (which was not certified by peer review) is the author/funder, who has granted bioRxiv a license to display the preprint in perpetuity. It is made available under aCC-BY-NC-ND 4.0 International license. 
Figure 1: Pds1 localizes to vacuoles independently of Autophagy

A. ${ }^{6 h r s ~ G A L-H O}$

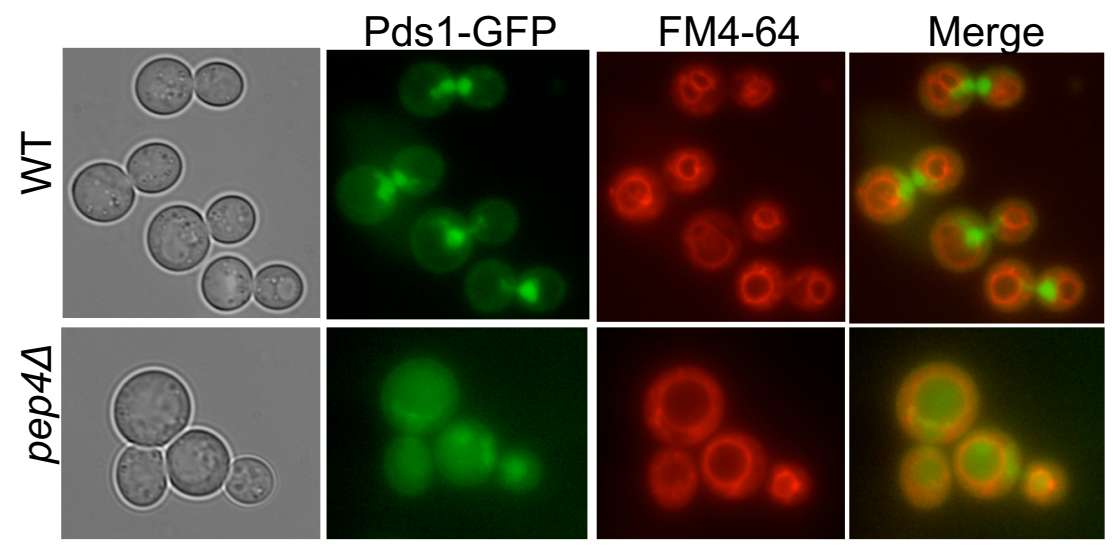

B.

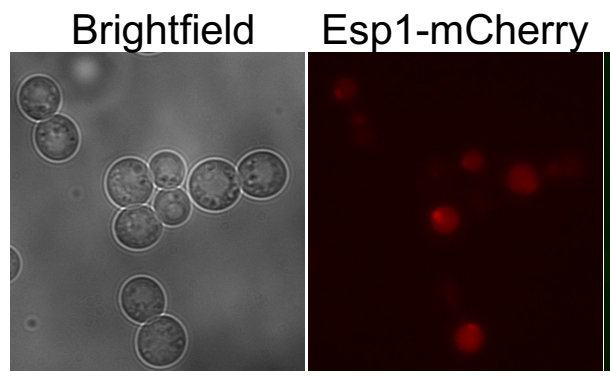

Pds1-GFP

Merge

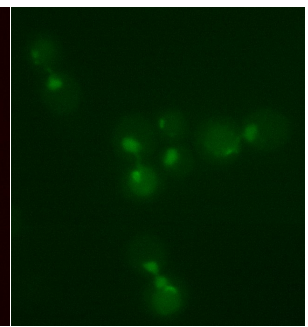

C.

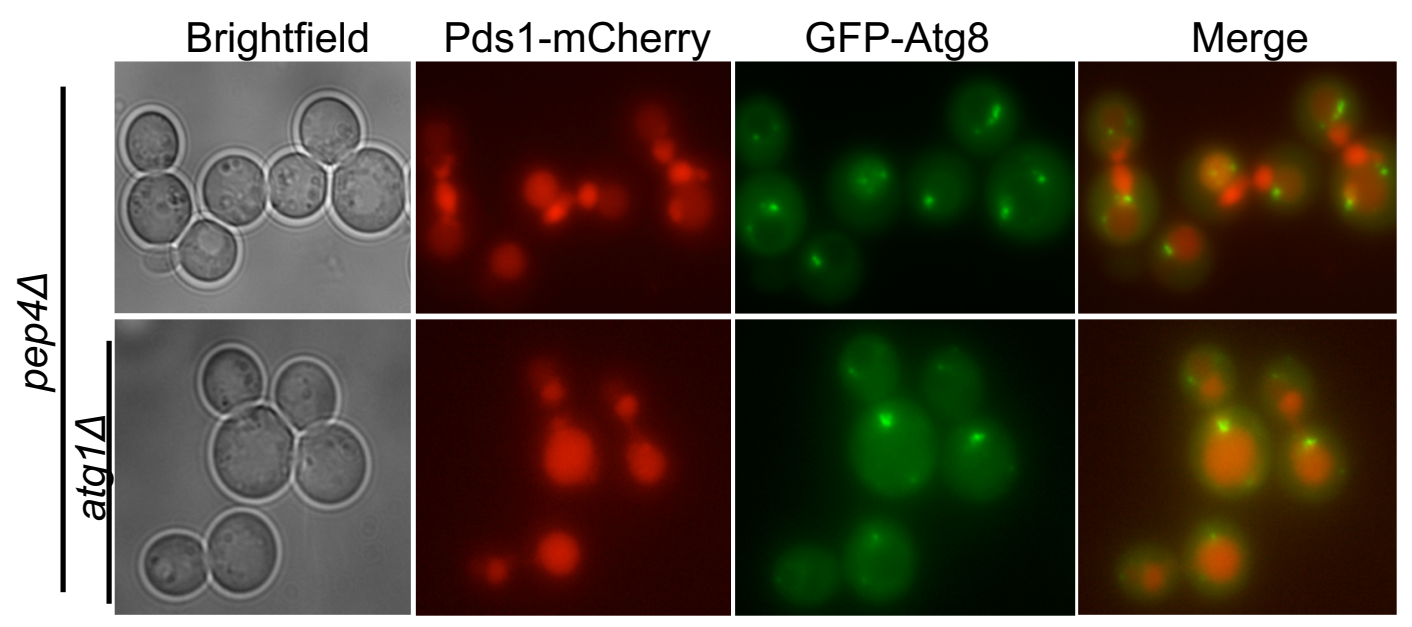

D.

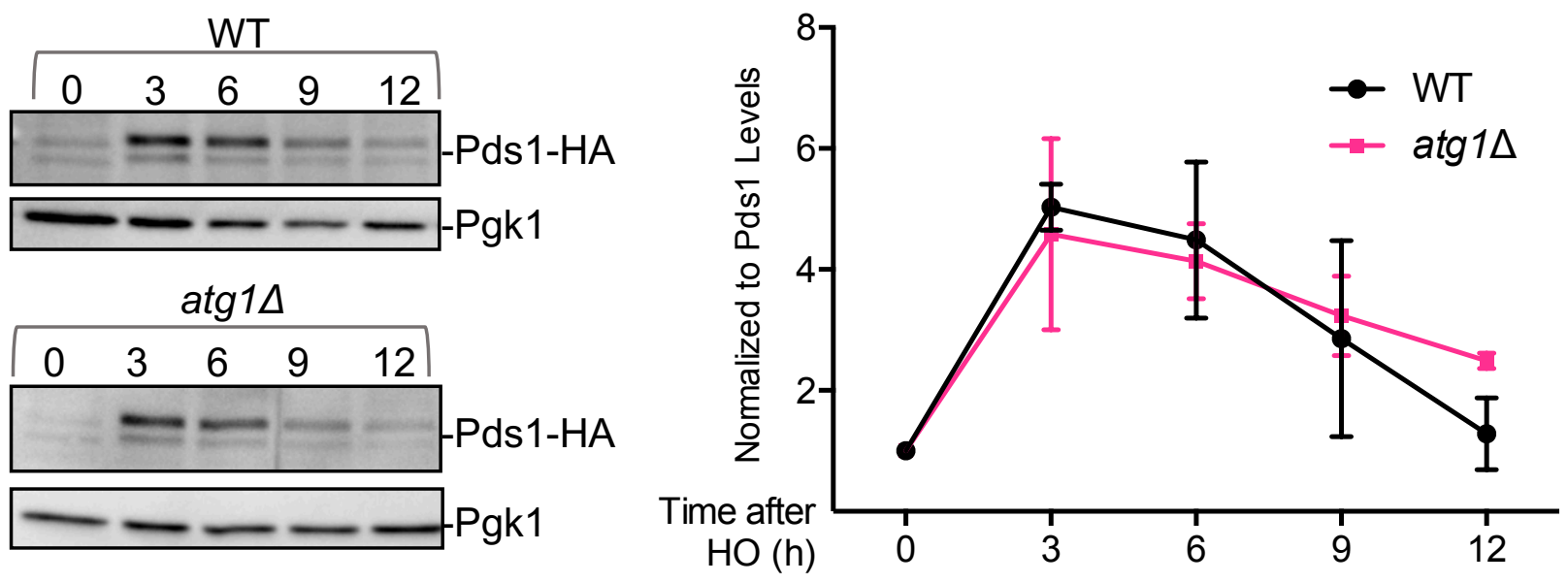


Figure 2: Pds1 localizes to vacuoles using components of the ALP pathway

A.
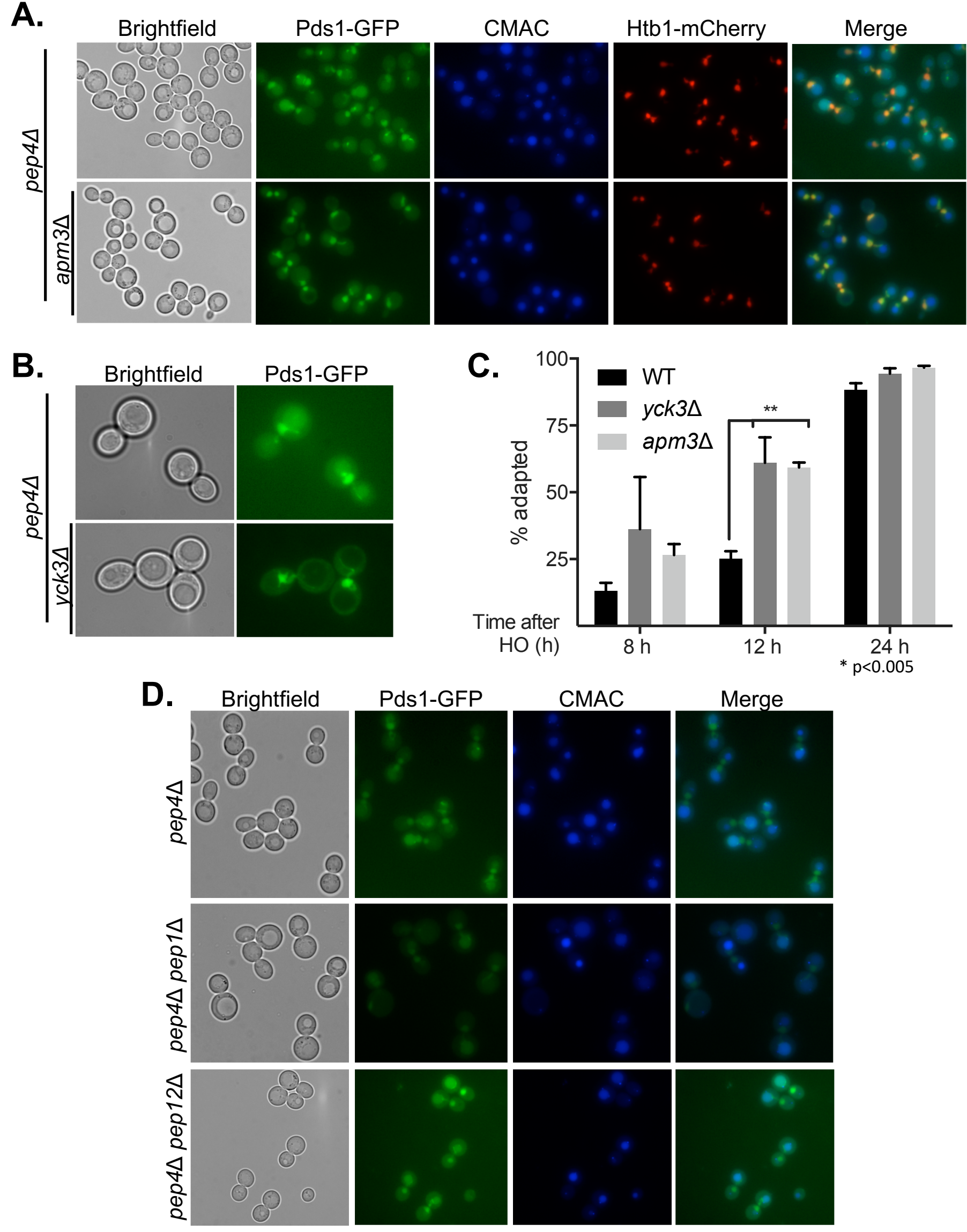
Figure 3: Pds1's localization and concentration in the absence of COPI and COPII vesicles

A.

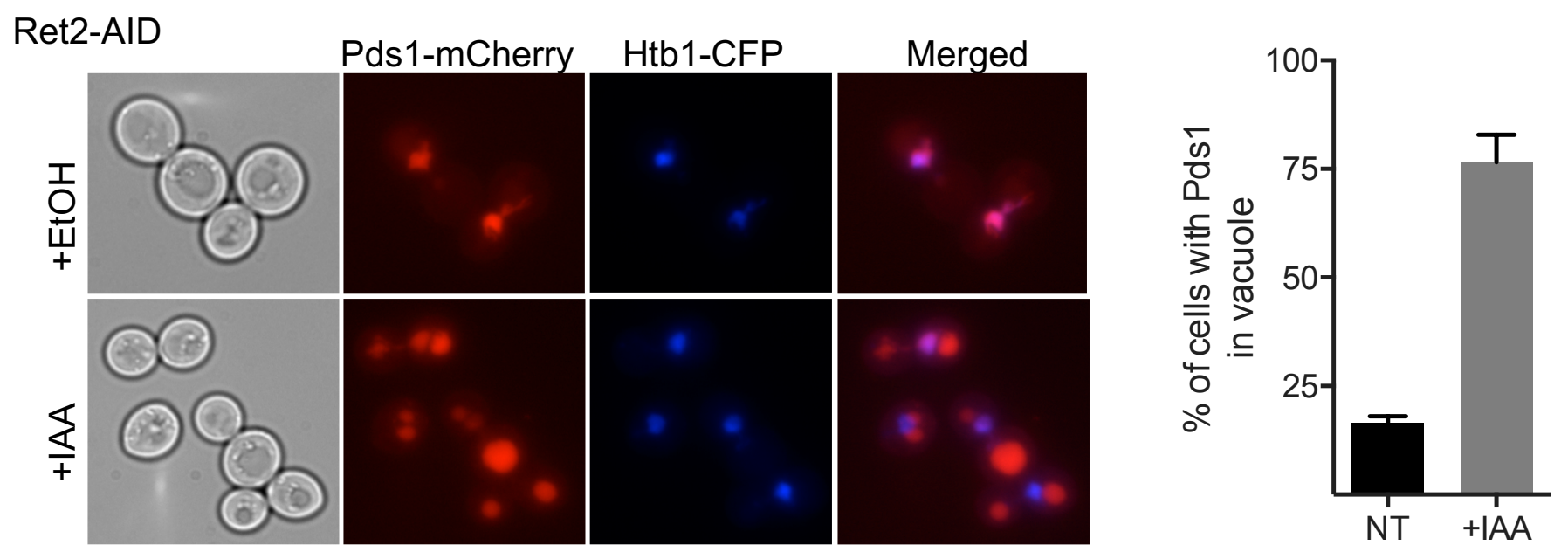

B. Sec23-AID
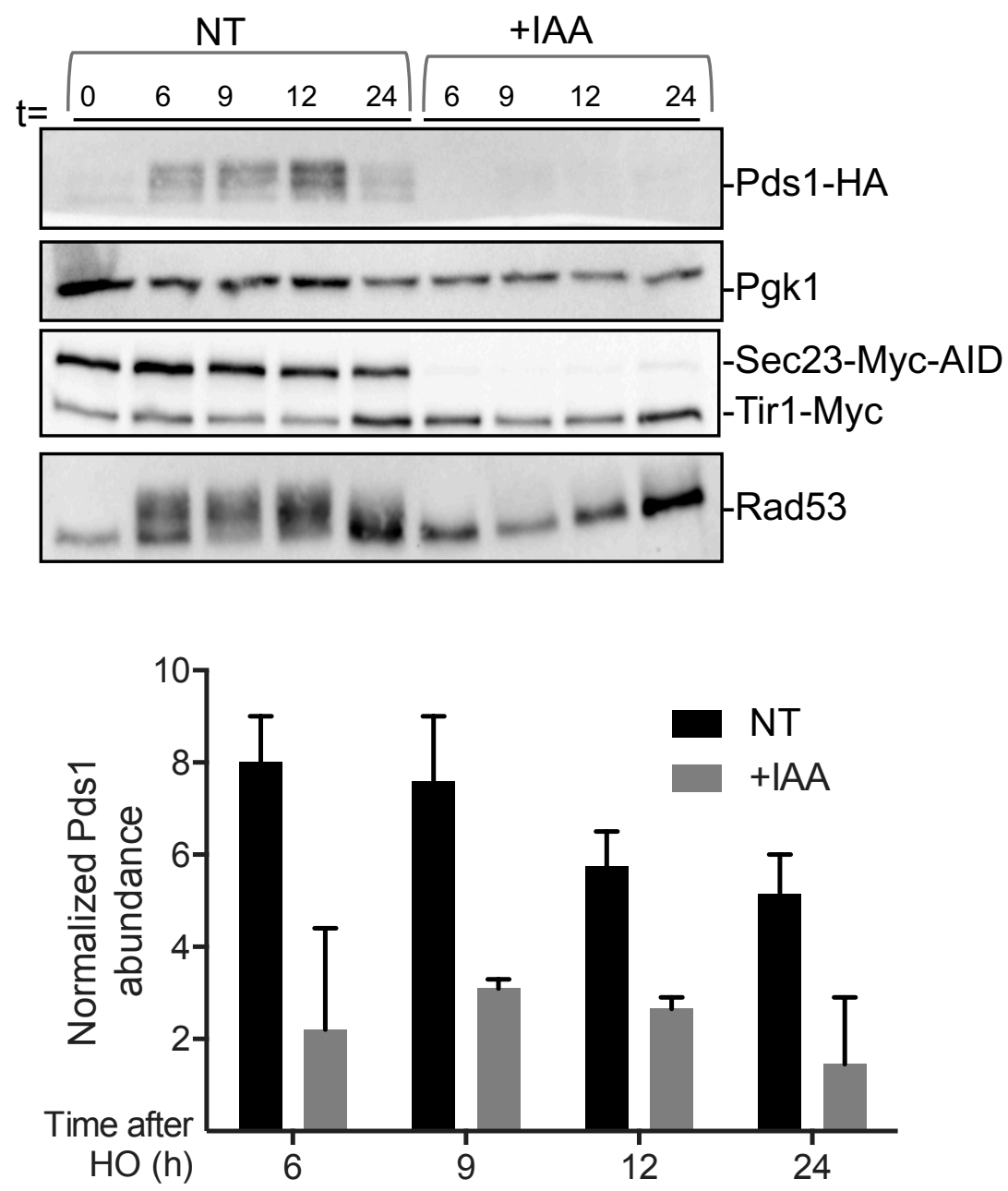
Figure 4: Effect of DTT on the DNA Damage Checkpoint

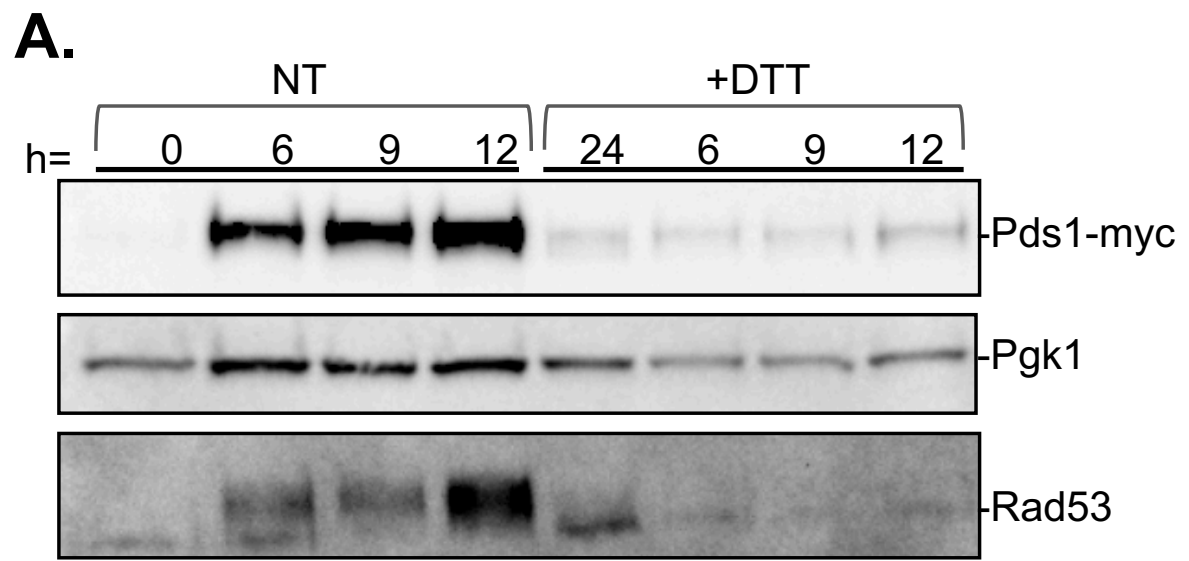

B.

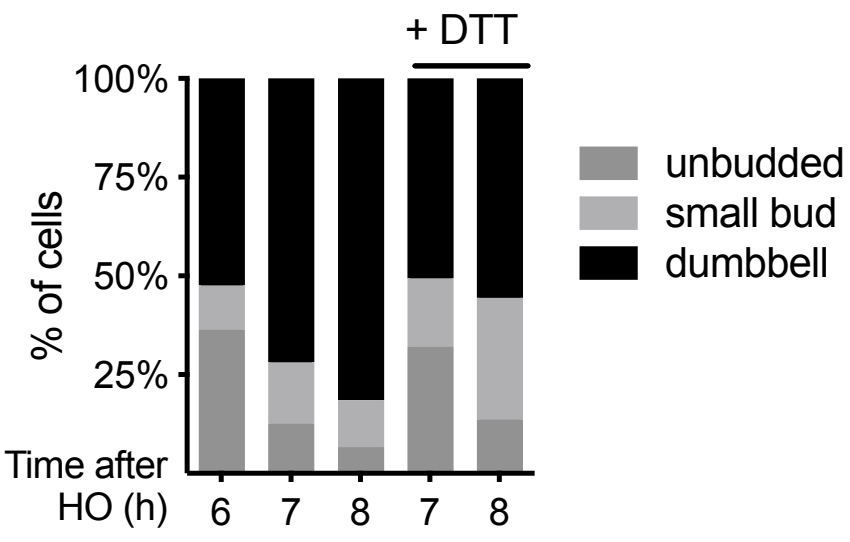

c.

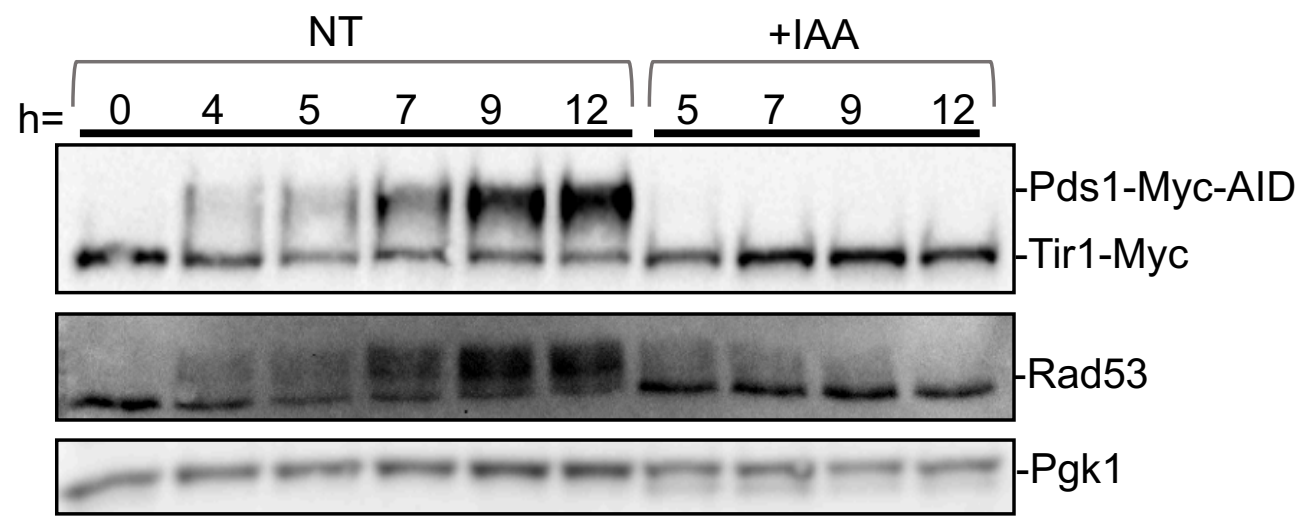


Figure 5: Crm1 is an exporter of Pds1 during the DNA damage response

A. $2 \%$ Galactose

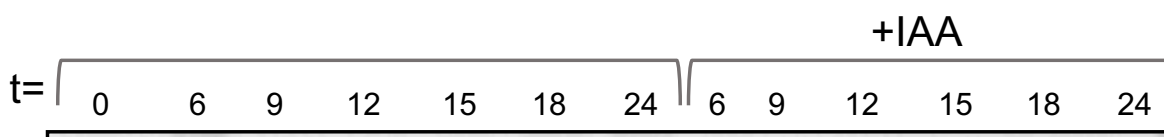

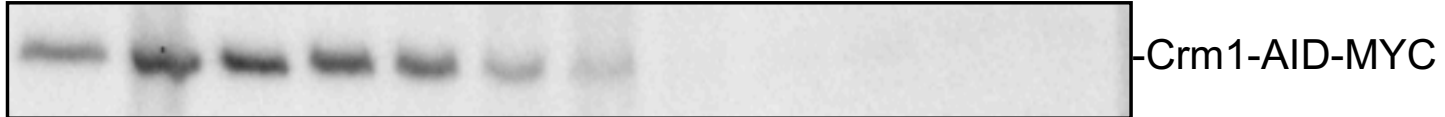

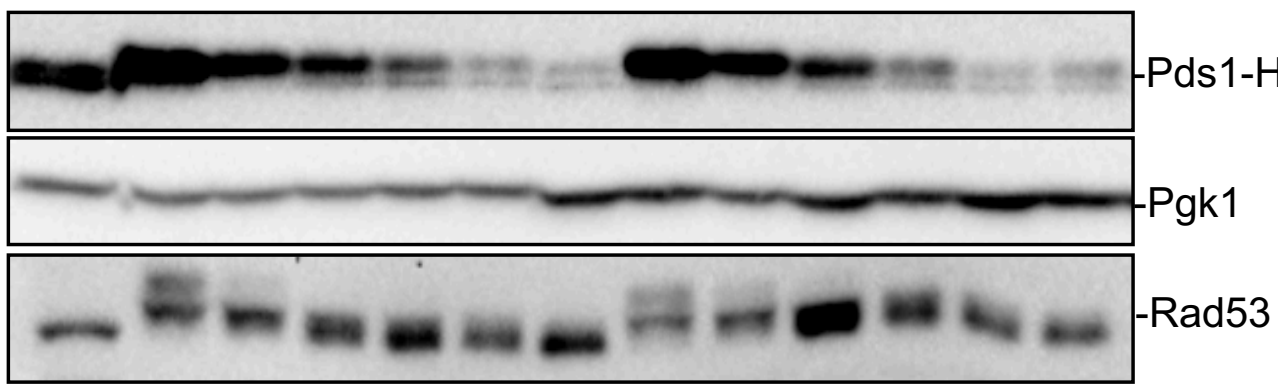

B.

2\% Galactose induced DNA Damage
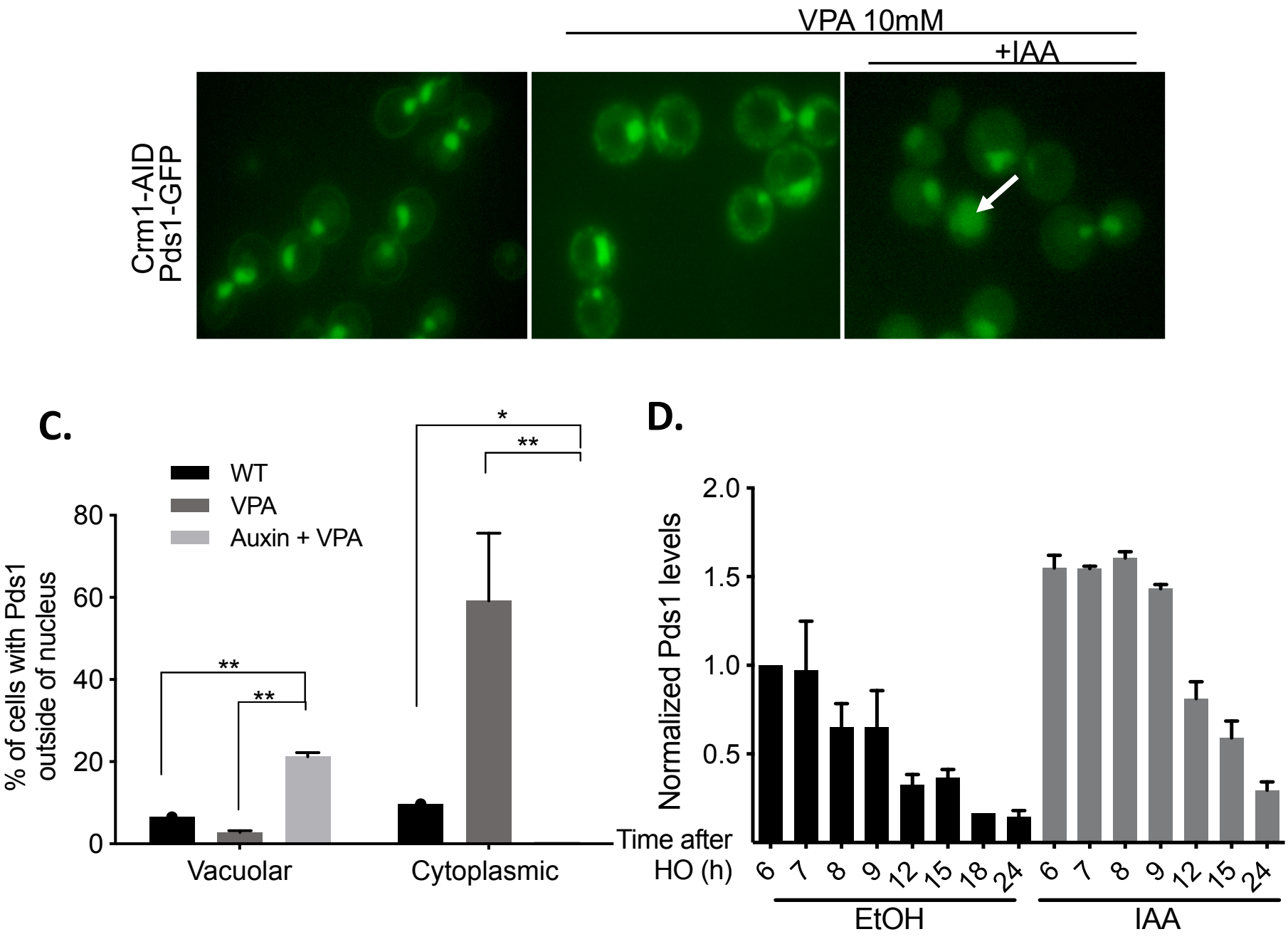
Supplemental Figure 1

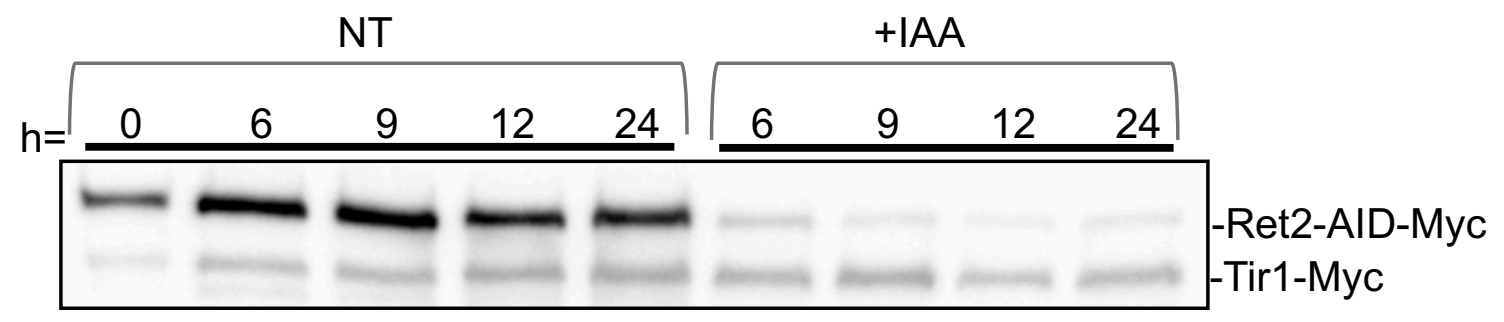

